Notfall Rettungsmed 2009 $\cdot 12: 489-490$

DOI 10.1007/s10049-009-1227-9

Online publiziert: 5. November 2009

(c) Springer Medizin Verlag 2009

\author{
U. Kreimeier ${ }^{1} \cdot$ H.-R. Arntz ${ }^{2}$ \\ ${ }^{1}$ Klinik für Anaesthesiologie, Klinikum der Ludwig-Maximilians-Universität, München \\ ${ }^{2}$ Medizinische Klinik II, Kardiologie und Pulmonologie, \\ Charité, Campus Benjamin Franklin, Berlin
}

\title{
Das gesamte Spektrum der Notfallmedizin
}

\section{Bedeutung der Fortführung der Rettungskette in die frühe klinische Versorgungsphase hinein}

\section{Liebe Leserinnen und Leser,}

neben präklinischen Fragestellungen werden in unserer Zeitschrift immer mehr Beiträge aus dem Bereich der Nahtstelle Rettungsdienst - Klinikaufnahme sowie innerklinische Notfälle veröffentlicht - eine Folge der Fortentwicklung der Notfallmedizin. Die Konzepte zur schnellen, adäquaten Versorgung der Patienten reichen vom revolutionären Ansatz des Heidelberger Klinomobil mit dem Motto:

„Der Arzt muss zum Patienten kommen und nicht der Patient zum Arzt"

über die Etablierung der Luftrettung in den 1970er Jahren, dem Ansatz der Sicherstellung von Perfusion und Restoration der Mikrozirkulation („small-volume resuscitation") sowie der frühen Intubation - speziell beim Schädel-Hirn- und Thoraxtrauma - bis zur Revision von „scoop and run“ hin $\mathrm{zu}$ „treat and run“. Bezeichnend ist die Einführung der Konzepte Advanced Trauma Life Support (ATLS ${ }^{\circ}$ ) und Prehospital Trauma Life Support (PHTLS ${ }^{\circ}$ ) mit dem Ziel der schnellen und genauen Einschätzung des Zustandes des Traumapatienten, der prioritätenorientierten Behandlung und der Entscheidung, ob die eigenen Ressourcen zur Behandlung des Patienten ausreichen oder ein Transfer des Patienten zu erwägen ist.

Besondere Bedeutung hat darüber hinaus in den letzten Jahrzehnten die Frühver- sorgung von nichttraumatologischen, insbesondere internistisch-kardiologischen Notfällen, gewonnen. Es hat sich daneben auch gezeigt, dass die von Ahnefeld aus Ulm beschriebene Rettungskette nicht am Eingang zur Klinik aufhört: Die Konzeption des zentralen Schockraums für polytraumatisierte Patienten oder auch der Aufbau von Chest Pain Units sind typische Elemente der Erweiterung der Rettungskette. Vielfältige neue diagnostische Möglichkeiten durch bildgebende Verfahren, die Sonographie und besonders die schnellen modernen Computertomographen haben dazu beigetragen, rascher gezielte Behandlungsstrategien zu ermöglichen.

Die ineinandergreifende Kette von präklinischer Versorgung und Erstmaßnahmen in der Notaufnahme gewinnt für die optimale Behandlung von Notfallpatienten zunehmend an Bedeutung, da in dieser Zeit die Schlüsselentscheidungen für die diagnostische und therapeutische Strategie fallen. Die im frühen Verlauf eingeleiteten Maßnahmen entscheiden tatsächlich wesentlich mit über das Outcome und damit über die Möglichkeiten der Reintegration des Patienten in das normale Leben. Der wichtige frühe Versorgungsablauf ist nicht nur gesundheitsökonomisch bedeutsam, sondern die im Zusammenhang entstehenden notfallmedizinischen Kosten für das Gesundheitssystem sind vor allem in Hinblick auf das gewünsch- te Ergebnis der Wiedereingliederung des Notfallpatienten in das normale selbstbestimmte Leben zu betrachten.

Mittlerweile werden 30-50\% aller stationären Patienten über die Notaufnahmen aufgenommen. Dies unterstreicht die zentrale Bedeutung dieser Entwicklung für die Prozesssteuerung einer Klinik unter qualitativen und ökonomischen Aspekten. $\mathrm{Ob}$ nun als zentrale oder interdisziplinäre Notaufnahme bezeichnet, hängt der Spezialisierungsgrad im Notaufnahmebereich wesentlich von den Versorgungsmöglichkeiten der vorhandenen und zu festgelegten Zeiten aufnahmebereiten Spezialabteilungen ab.

\section{() Von allen stationären \\ Patienten kommen 30-50\% über die Notaufnahmen}

„Notfall + Rettungsmedizin“ begleitet diese Entwicklung durch Leitthemen, Übersichtsarbeiten, Weiterbildungsbeiträge und eine zunehmende Anzahl von Originalien und Kasuistiken, die den aktuellen wissenschaftlichen Erkenntnisstand widerspiegeln. Als offizielles Organ der Deutschen Interdisziplinären Vereinigung für Intensiv- und Notfallmedizin (DIVI), des Deutschen Rats für Wiederbelebung - German Resuscitation Council (GRC) e.V. und des Austrian Resuscitation Council (ARC) bieten wir Ihnen, liebe Leserinnen und Leser, 
Informationen aus erster Hand. Die Veröffentlichung von Stellungnahmen der Fachgesellschaften und von Leitlinien ist fester Bestandteil des Konzepts unserer Zeitschrift, zusammen mit neuesten wissenschaftlichen Erkenntnissen, State of the Art Reviews und der Vorstellung neuer Leitlinien. Wir möchten Sie über sämtliche Teilbereiche der Notfallmedizin informieren: die präklinische Phase, klinische Aufnahme- als auch frühe klinische Versorgungsphase, ergänzt durch innerklinische Notfälle und deren Prävention. Das gesamte Spektrum der Notfallmedizin eben...

\section{Mit den besten Grüßen}

Ihre

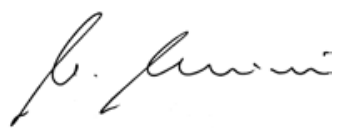

U. Kreimeier

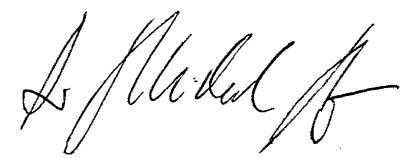

H.-R. Arntz

\section{Korrespondenzadresse}

\section{Prof. Dr. U. Kreimeier}

Klinik für Anästhesiologie, Klinikum der Universität München

Nussbaumstr. 20, 80336 München uwe.kreimeier@med.uni-muenchen.de
„Notfall + Rettungsmedizin“ bietet Ihnen 8-mal im Jahr umfassende und aktuelle Beiträge zu interessanten Themenschwerpunkten (Leitthemen) aus allen Bereichen der Notfallmedizin.

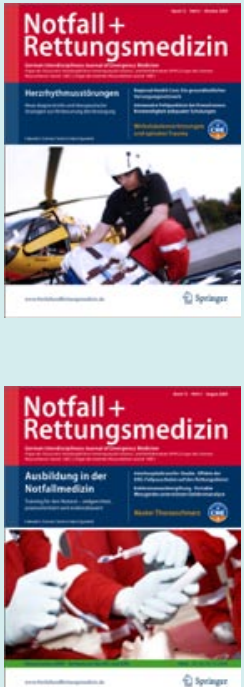

2008

- Heft 01/08 Evidenzbasierte Notfallmedizin

- Heft 02/08 ERC-Leitlinien 2005 - 2 Jahre danach

- Heft 03/08 Schlaganfall

- Heft 04/08 Luftrettung

- Heft 05/08 Invasive Notfalltechniken

- Heft 06/08 Polytrauma

- Heft 07/08 Postresuscitation Care

- Heft 08/08 Der psychiatrische Notfall

2009

- Heft 01/09 Die soziale Dimension der Notfallmedizin

- Heft 02/09 Synkope und Schwindel

- Heft 03/09 Volumentherapie in der Notfallmedizin

- Heft 04/09 Interdisziplinäre Notaufnahme

- Heft 05/09 Ausbildung in der Notfallmedizin

- Heft 06/09 Lebensbedrohliche Blutungen

- Heft 07/09 Herzrhythmusstörungen

- Heft 08/09 Notfälle beim Kind

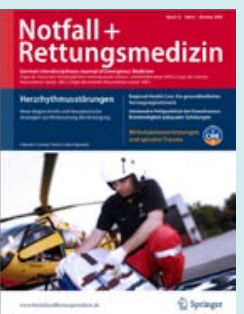

Alle Beiträge auf einen Blick

Abonnenten haben online Zugriff auf alle Beiträge im elektronischen Volltextarchiv unter

\section{www.NotfallundRettungsmedizin.de}

Ins Volltextarchiv gelangen Sie von der Startseite über den Navigationspunkt "Online-Archiv".

Eine interessante Lektüre wünscht Ihnen

Ihre Redaktion „Notfall + Rettungsmedizin” 